\title{
ADICIONES A LAS ALGAS DE AGUA DULCE DE LAS CASCADAS DE BARRANCO
}

César Acleto 0.

Departamento de Botánica del Museo de Historia Natural "Javier Prado" de la Universidad Nacional Mayar de San Marcos, Lima, Perú.

S U M A R I O

En el presente trabajo se describen 13 especies de algas de agua dulce, colectodas en las Cascadas de Barranco, Departamento de Lima. Las especies estudiadas constituyen nuevos registros poro la zona y 8 de ellas lo son para la flora peruana.

\section{S U M M A R Y}

The present poper reports the description of Thirteen species of freshwoter olgoe collected ot the Coscadas of Barranco, Department of Lima. All of them constitutes the first record for the studied area and Eight are news for the Peruvion floro.

\section{INTRODUCION}

En una contribución anterior, el autor (1966), describió gran parte de las algas de agua dulce de las Cascadas de Barranco. El presente trabajo se refiere a los nuevos registros de algas determinadas en las colecciones anteriormente estudiadas y en las efectuadas con posterioridad en dicha zona.

Se describen 13 especies correspondientes a los siguientes grupos: CYANOPHYTA (7): Coelosphaerium kuetzingianun Naegeli, Dermocarpa versicolor (Borzi) Geitler, Oscillatoria splendida Greville ex Gomont, Oscillatoria pseudogeminata G. Schmid, Microcoleus chthonoplastes (Mertens) Thuret, Scytonema hoffmannii Agardh ex Gomont et Flah. y Dichothrix baueriana (Grun.) Born. et Flah.; XANTHOPHYCEAE (1): Vaucheria geminata (Vaucher) DC; CHLOROPHYTA (3): Stigeoclonium lubricum (Dillw.) Kützing, Spirogyra decimina (Müller) Kützing y Closterium pseudolunula Borge; y RHODOPHYTA (2): Compsopogon coeruleus (Balbis) Mont., y Rhodochorton venezuelensis $D^{\prime}$ Lacoste et Ganesan.

\section{MATERIAL Y METODOS}

El material estudiado fue colectado en la zona indicada y está preservado en una solución de formol al $5 \%$ y se conserva en la Sección Algas del Herbario
San Marcos (USM) del Museo de Historia Natural "Javier Prado".

Las observaciones microscópicas han sido realizadas en preparados temporales del material fijado, incluídos en una gota de glicerina diluída o en el jarabe de azúcar KARO ambos en una solución al $50 \%$. Se utilizó también anilina azul al $1 \%$ con el objeto de diferenciar mejor las estructuras.

Los dibujos y las microfotografías que ilustran este trabajo han sido realizados por el autor, empleando la cómara clara y película en blanco y negro Kodak Plus $X$ respectivamente.

\section{PARTE SISTEMATICA}

\section{DESCRIPCION DE LAS ESPECIES}

\section{CYANOPHYTA}

\section{CHROOCOCCALES \\ Familia Chroococcaceae \\ COELOSPHAERIUM Naegeli, 1844 \\ Coelosphaerium Kuetzingianum Naegeli \\ Fig. 1}

Colonia, libre, esférica o ligeramente oblonga, de 15.6 a 85.1 micras de diámetro; cubierta colonial mucilaginosa, firme a difluente, homogénea y hialina; células esféricas o subesféricas, de 3 a 4 micras de diómetro, dispuestas irregularmente próximas a la periferie de la cubierta colonial; protoplasto verde lúteo a 
verde oliváceo brillante, con granulaciones pequeñas y escasas.

Esta especie fue descrita anteriormente como Gomphosphaeria sp. Acleto $(1966$, p. 35 , figs. 14 y 15), y es considerada como Gomphosphaeria lacustris Chodat según Drouet (1956, P. 96, figs. 175 y 176). Los especímenes registrados en el área estudiada no tienen las características de esta última, pues las células no se ubican en los extremos de hilos citoplasmáticos radiales, tal cual se indica para dicha especie.

Material estudiado: C. Acleto A-39.

DERMOCARPALES

Familia Dermocarpaceae

DERMOCARPA Crouan, 1858

Dermocarpa versicolor (Borzi) Geitler

Figs. 2 y 30

Células ovoides; generalmente gregarias, alcanzan hasta 15 micras de diámetro; protoplasto verde azul brillante; esporangios con 4 a 8 endosporas, se liberan a través del ápice, algunas permanecen dentro del esporangio casi vacío.

Esta especie ha sido observada epífita en las células basales de Rhodochorton venezuelensis.

Material estudiado: C. Acleto A-260.

HORMOGONALES

Familia Oscillatoriaceae

OSCILLATORIA Vaucher, 1803

Oscillatoria splendida Greville ex Gomont Fig. 3

Tricomas rectos o curvados, azul-verde o verde olivo; no constrictos a nivel de la pared transversal, atenuados progresivamente hacia el ápice; células cilíndricas de 2.4 micras de diámetro $y$ 5.4 a 6 micras de longitud, con granulaciones a nivel de las septas; extremo más - menos curvado, algunas veces semeja una hoz o-segadora; célula apical capitada, redondeada, sin caliptra.

Forman masas flotantes con otras spp. de Oscillatoria.

Drouet (1968, p. 166) considera a esta especie como basónimo de Porphyrosiphon splendidus Greville.
Material estudiado: C. Acleto A-329.

Oscillatoria pseudogeminata G. Schmid Fig. 4

Tricomas rectos, verde claro, constrictos a nivel de la pared transversal, curvados en sus extremos, no atenuados; células generalmente de 6 a 6.3 micras de diámetro, de igual longitud o algo más largas o cortas que anchas, con finas granulaciones; célula apical redondeada, sin caliptra.

Forma natas flotantes entre gramíneas.

Drouet (1968, p. 57) incluye a esta especie entre los ecofenos de Schizothrix calcicola (Agardh) Gomont.

Material estudiado: C. Acleto A-328.

MICROCOLEUS Desmazieres, 1823 Microcoleus chthonoplastes (Mertens)

Thuret

Figs. 5,6 y 31

Filamentos simples, a veces ramificados, con los extremos atenuados, de color verde azul brillante; estuche homogéneo, hialino, de espesor variado y superficie irregular, frecuentemente con los extremos abiertos en los filamentos adultos; tricomas numerosos dispuestos apretadamente, en la parte media del filamento, disminuyen hacia los extremos, verde azul intenso, constrictos a nivel de la pared transversal; células cilíndricas de 2.5 a 6 micras de diámetro por 3.6 a $11 \mathrm{mi}$ cras de longitud, sin granulaciones a lo largo de las paredes transversales; protoplasto finamente granulado; célula apical cónica, de extremo terminado en punta, no capitada.

Habita sobre terreno húmedo protegido, en los bordes del canal principal.

Material estudiado: C. Acleto A-318.

Familia Scytonemataceae

SCYTONEMA Agardh, 1824

Scytonema hoffmannii Agardh ex Gomont et Flah.

Figs. 7, 8, 9 y 34

Filamentos entrecruzados, plomizo azulado, impregnados con carbonato de 
calcio, de 7 a 14 micras de diámetro, en fascículos, con falsas ramas, generalmente en pares; estuche firme, estratificado, pardo amarillento o hialino; tricoma verde azul a verde olivo, sin constricción a nivel de la pared transversal; células cilindricas de 5 a 12 micras de diámetro; protoplasto homogéneo.

Habita en los ambientes húmedos subaéreos, formando un estrato afelpado, plomizo, azulado.

Material estudiado: C. Acleto A-338.

DICHOTHRIX Zanardini, 1858

Dichothrix baueriana (Gru.) Born. et Flah. Figs. $10,11,12,32$ y 33

Planta cespitosa, expandida, alcanza $4 \mathrm{~mm}$. de altura, marrón o verde oscuro; filamentos flexuosos de 15 a 18 micras de diámetro, atenuados hacia el ápice, con falsas ramas subdicótomas, generalmente con 2 ó 3 tricomas en un estuche común en la región basal, con un solo tricoma en las regiones media y distal; estuche homogéneo, hialino o estratificado, marrón amarillento; tricomas de 5 a 9 micras de diámetro, azul verde pálido, constrictas a nivel de la pared transversal, terminan gradualmente en un pelo hialino; células tan largas o $1 / 2$ más largas que anchas; protoplasto granular; heterocisto basal, subesférico.

Forma un tapiz afelpado de aspecto característico.

Material estudiado: C. Acleto A-281.

\section{CHRYSOPHYTA}

\section{XANTHOPHYCEAE}

HETEROSIPHONALES

Familia Vaucheriaceae

VAUCHERIA DC., 1803

Vaucheria geminata (Vaucher) DC.

Figs. 13, 14, 35 y 36

Filamentos ramificados, entrecruzados, verde amarillento brillante, de 81 a 108 micras de diámetro, con numerosos cromatóforos discoidales, escasos en la porción basal; oogonios generalmente en pares, globosos o subglobosos de $94 \mathrm{mi}$ cras de diómetro, localizados en el extremo de una rama corta constricta en su base al nivel del eje principal del cual deriva; anteridio curvado o circinado, situado en el extremo distal entre los oogonios.

Frecuentemente forma una mata suave afelpada en agua corriente, sin estructuras de reproducción en este habitat. Los oogonios y anteridios se forman sólo cuando los filamentos emergen parcialmente hacia suelos húmedos.

Material estudiado: C. Acleto A-62,

C. Acleto A-271

\section{CHLOROPHYTA}

ULOTRICHALES

Familia Ulotrichaceae

STIGEOCLONIUM Kützing, 1843

Stigeoclonium lubricum (Dillw.) Kützing Figs. 15,16 y 17

Planta filamentosa, verde brillante de aspecto mucoso, diferenciada en una porción postrada, reducida y una porción erguida muy desarrollada, con ramas generalmente alternas a veces, verticiladas u opuestas forman fascículos en la parte terminal; las células basales del eje principal diferenciadas en un rizoide ramificado, irregular; células de los filamentos principales y ramas en forma de barril, miden 12 micras de diámetro por 18 a 30 micras de longitud, progresivamente más pequeñas en las ramas; célula apical de extremo agudo o cónico, mide hasta 6 micras de diómetro; cloroplasto laminar, parietal ecuatorial.

Esta especie habita sobre cantos rodados en agua de corriente rápida, forma un tapiz suave, verde brillante.

Material estudiado: C. Acleto A-234

\section{CONJUGALES}

Familia Zygnemataceae

SPIROGYRA Link, 1820

Spirogyra decimina (Müller) Kützing

Figs. $18,19,37$ y 38

Planta filamentosa, de color verde brillante; células cilíndricas de $40.5 \mathrm{mi}$ cras de diámetro por 54 a 135 micras de longitud, con dos cloroplastos cintados, planos, de ancho irregular, más amplio a nivel de los pirenoides axiales, dan 2 vueltas regulares cada uno. Conjugación 
escaleriforme, frecuentemente entre los gametangios de dos filamentos paralelos o entre los gametangios de un filamento central con los de dos filamentos laterales; zigospora ovoide, de 39 a 42 micras de diámetro por 51 a 63 micras de longitud, de pared moderadamente engrosada, más notorio en los extremos, amarillenta, sin ornamentación visible.

Forman natas flotantes en los canales con agua de circulación lenta.

Material estudiado: C. Acleto A-266.

Familia Desmidiaceae

CLOSTERIUM Nitzsch, 1817

Closterium pseudolunula Borge

Fig. 20

Células grandes de 297 a 540 micras de longitud por 42 a 63 micras de diámetro, fusiformes, de margen externo ligeramente curvado, el interno plano, ápice redondeado; pared celular lisa, ligeramente amarilla; con dos cloroplastos, estriados longitudinalmente, cada uno con 10 a 14 pirenoides axiales.

Frecuente en el fondo lodoso de aguas estancadas, o en charcas superficiales.

Material estudiado: C. Acleto A-6, A-7 y A-8.

\section{RHODOPHYTA}

BANGIOPHYCIDAE

COMPSOGONALES

Familia Bangiophycidae

COMPSOPOGON Montagne, 1850

Compsopogon coeruleus (Balbis) Mont.

Figs. 21, 22, 23, 24, 25 y 26

Planta filamentosa, verde o marrón violácea, alcanza hasta $30 \mathrm{~cm}$. de altura por 1.5 a $2 \mathrm{~mm}$. de diámetro en la parte más ancha del eje principal. Ramas alternas, libres, hasta de 5 órdenes, generalmente uniseriadas cuando jóvenes o en su extremo distal; con células discoidales a cilíndricas de 21 a 24 micras de diámetro; la célula apical con el extremo libre redondeado; las porciones más viejas con un eje axial de células grandes, cubierta por células corticales de contorno poligonal en vista superficial de 45 micras de diámetro; cromatóforos numerosos, alar- gados o curvados, parietales, irregularmente dispuestos. Monosporas originadas por división parcial de las células corticales del filamento principal, o de las células de las ramas uniseriadas; miden 12 a 21 micras de diámetro.

Los ejemplares colectados en las Cascadas de Barranco fueron encontrados flotando libremente en el borde del canal de agua corriente lenta. En la mayoría de los filamentos más desarrollados, no fue posible distinguir el rizoide, sin embargo, en los ejemplares jóvenes, que crecen epifiticamente sobre otras, el rizoide es expandido y está constituído por las células basales uniseriadas del filamento principal y por las ramas uniseriadas que derivan de ellas y crecen adheridas hacia abajo.

Material estudiado: C. Acleto A-342.

FLORIDIOPHYCIDAE

NEMALIALES

Familia Acrochaetiaceae

RHODOCHORTON Naegeli, 1861

Rhodochorton venezuelensis 'D'Lacoste et Ganesan.

Figs. 27, 28, 29, 39 y 40

Plantas heterotricas, pequeñas, alcanzan $1.5 \mathrm{~mm}$. de altura, de color verde rojizo, o marrón rojizo; la porción basal de cada filamento consta de pocas células cilíndricas, irregulares, anastomosadas con las de los otros filamentos formando una capa contínua; la porción erguida consiste de filamentos uniseriados, con ramificaciones alternas hasta de 3 órdenes, más abundantes en la parte media y superior; células del filamento principal cilíndricas, de 12 a 18 micras de diámetro por 39 a 87 micras de longitud; algunos filamentos más desarrollados, las células inferiores presentan rizoides pluricelulares, orientados hacia la parte basal; las células de las ramas miden 9 a 12 micras de diámetro por 27 a 51 micras de longitud; las apicales miden 9 a 10.5 micras de diámetro por 60 a 90 micras de longitud; cromatóforos pequeños, numerosos, redondeados o ligeramente alargados, más compactos en las células apicales; conexiones citoplasmáticas visibles teñidas con anilina azul a) $1 \%$; monosporangios ovoides, de 13.5 micras de diámetro por 15 a 18 micras de longitud, localizadas en el extremo de 
las ramas laterales cortas, solitarios o en grupos.

Forman un tapiz verde rojizo, suave, en la superficie libre de pequeños cantos rodados sumergidos en agua de corriente lenta.

Esta especie la describí (1966, p. 82, microf. G) como la forma juvenil o la fase "Chantransia" de Batrachospermum sp. Posteriormente he comprobado que corresponde al género Rhodochorton $y$ con mucha similitud a Rhodochorton venezuelensis D'Lacoste et Ganesan, especie descrita para Venezuela y de la cual difiere sólo por su menor tamaño, en hábito, células vegetativas $\mathrm{y}$ monosporangios.

\section{Dimensiones}

Planta ..... $4 \mathrm{~mm}$.

Células del fila-

mento principal 11-37 micras por 51-110 micras

Cél. de las ramas 10-22 micras por 28-55 micras

Célula apical . 10-20 micras por 45-118 micras

Monosporangios

\section{Ejemplares de Perú}

$1.5 \mathrm{~mm}$.

12-18 micras por 39-87 micras

9-12 micras por 27-51 micras

9-10.5 micras por 60-90 micras 13 micras por 15-18 micras
Geográficamente este es el primer registro de la especie para el hemisferio sur de Sudamérica.

Material estudiado: E. Cerrate y L. Sánchez s.n., C. Acleto A-260.

\section{AGRADECIMIENTOS}

Expreso mi agradecimiento al Dr. Francis Drouet de la Academy of Natural Sciences of Philadelphia, U.S.A. por la determinación de algunas especies de CYANOPHYTA descritas en esta contribución.

\section{REFERENCIAS BIBLIOGRAFICAS}

ACLETO, O. C. - 1966. Algas de agua dulce de las Coscodas de Barranco. Publ. Mus. Hist. Not. "Javier Prodo", Serie B. 21:1-65.

ACLETO, O. C. - 1969. Dos especies de Cyanophyta nuevas que se registran para el Perú. Pub. Mus. Hist. Not. "Javier Prado", Serie B. 23:1-8.

DESIKACHARY, T. V. - 1959. Cynophyto. Indian Council of Agricultural Research, 686 pp., 139 pls., New Delhi
D'LACOSTE, L. G. and E. K. GANESAN - 1972 A new freshwater species of Rhodochorton (Rhodophyto, Nemaliales) from Venezuela. Phycologia, 11:233-278, 10 figs.

DROUET, F. and W. A. DAILY - 1956. Revision of the Coccoid Myxophyceoe. Butler Univ. Bot. Studies, 12:1-128, 377 figs.

DROUET, F. - 1968. Revision of the clasificotion of the Oscillatorioceae. The Acod. of Notu. Sci. of Philadelphia, Monograph 15:1-370 131 figs.

GOMONT, M. - 1892. Monogrophie des Oscillariees (Nostocees Homocystees) Ann. Sci. Natu. 7e. Ser. Bot. 15:263-368, Lam. 6-14; 91-264, Lom. 1-7, Poris. Reimp. 1. Cromer Weinheim, 1962. Alemonic.

HALPERIN, Delio R. de - 1967. Cionoficeos Morinas de Puerto Deseado (Argentina). 11. Dar winiona 14: 273-354, 14 lám.

OLIVEIRA FILHO, E. C. de e S. M. B. PEREIRA 1973. Notos sobre o género Compsopogon Montogne (Rhodophyta - Copsopogonoceoe) in Brazil. Bol. de Botónica 1:85-94.

PRESCOTT, G. W. .- 1962. Algoe of the Western Great Lokes, Rev. ed. WM. C. Brown Co. Pub., Dubuque, lowa

PUJALS, Carmen - 1967. Presencia en la Argentina del género Compsopogon (Rhodophyto, Bongiophycidae) Com. Mus. Arg. Cienc. Nat "Bernordino Rivadavia" 1:47-53, 2 lám. 
SMITH, G. M. - 1950. Fresh-water algoe of the United States. 2nd. ed. MeGraw-Hill Book Co. New York.

TAFT, C. E. and Celeste W. TAFT - 1971. The algoe of Western Lake Erie. Bull. Ohio Biol. Survey, New Ser., 4:1-189.

TILDEN, Josephine - 1910. Minnesoto Algoe. I. The Myxophyceae of the North America and odjocent region including Central America,
Greenland, Bermudo, the West Indies and Howai, iv + 328 pp., 20 pls.

VENKATARAMAN, G. S. - 1961. Vaucherioceoe Indian Council of Agric. Reseorch, $112 \mathrm{pp}$. 74 figs. New Delhi.

ZUNIIGA, A. Reino - 1972. Algas frecuentes en la Planto de Tratomiento de aguo potable "Gustavo Lourie Solis" de la Atarjea, Lima, Raymondiano 5:103-121. 


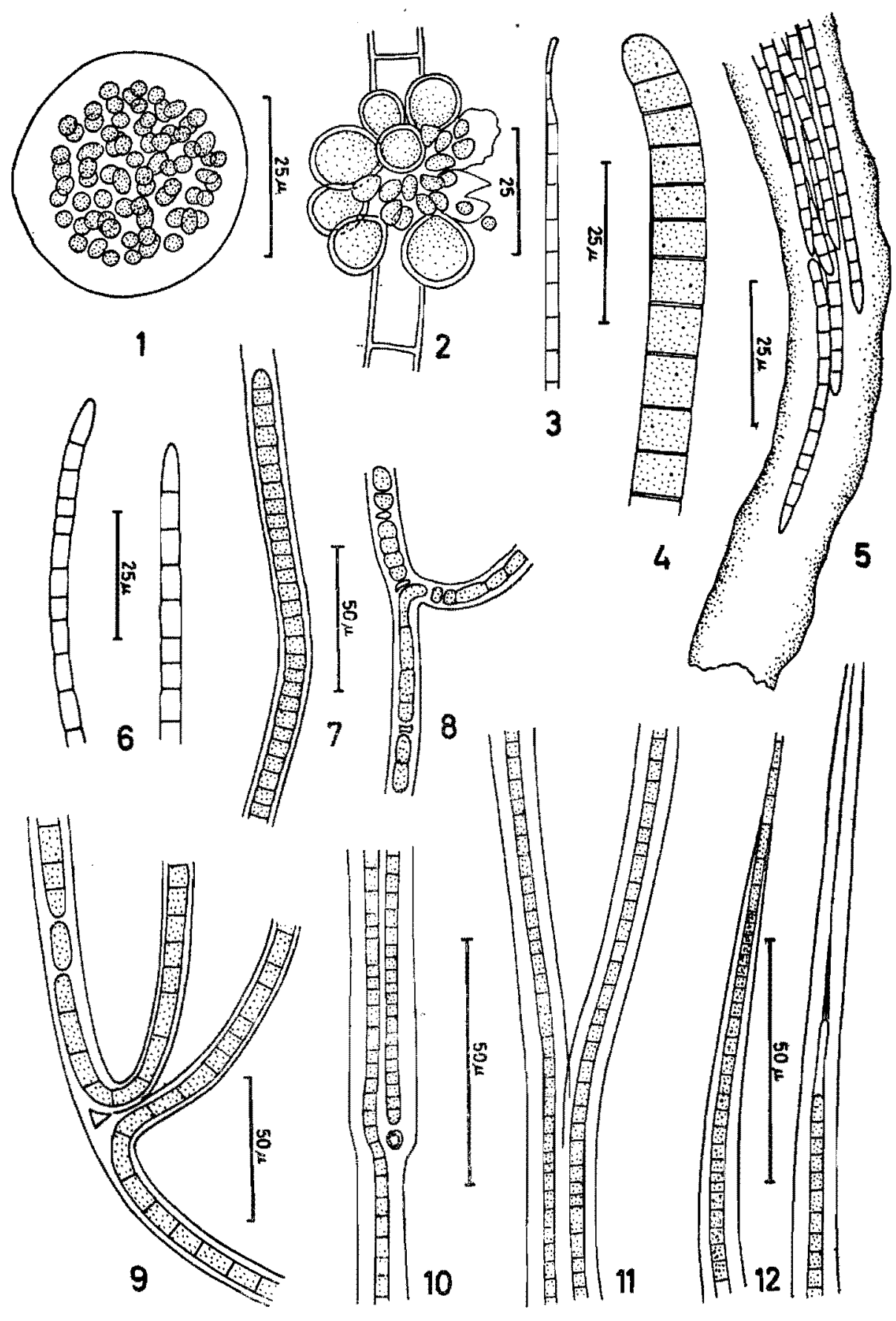

Fig. 1 Coelosphoerium kuetzingianum Noegell,- Fig. 2 Dermocarpa versicolor (Borzi) Geitler, individuos gregorios, epifitos en Rhodochorton venezuelensis.- Fig. 3 Oscillatoria splendida Greville ex Gomont, extremo terminal de un tricoma.-Fig. 4 Oscillotoria pseudogeminata $G$. Schmid, porción terminal de un tricoma.- Figs. 5 y 6 Microcoleus chthonoplastes (Mertens) Thuret. (Fig. 5) Porte terminal de un filamento. (Fig 6) Corocteristicas de dos tricomas individuales.- Figs. 7, 8 y 9 Scytonema hotfmannii Agardh ex Gomont et Flah., caracteristicas del filamento y las ramificaciones.- Figs. 10, 11 y 12 Dichothrix baveriana (Grud.) Born. et Flah. (Fig. 10) Porción media de un filamento conteniendo dos tricomas. (Figs. 11 y 12) Coracteristicas del extremo terminal del filomento. 


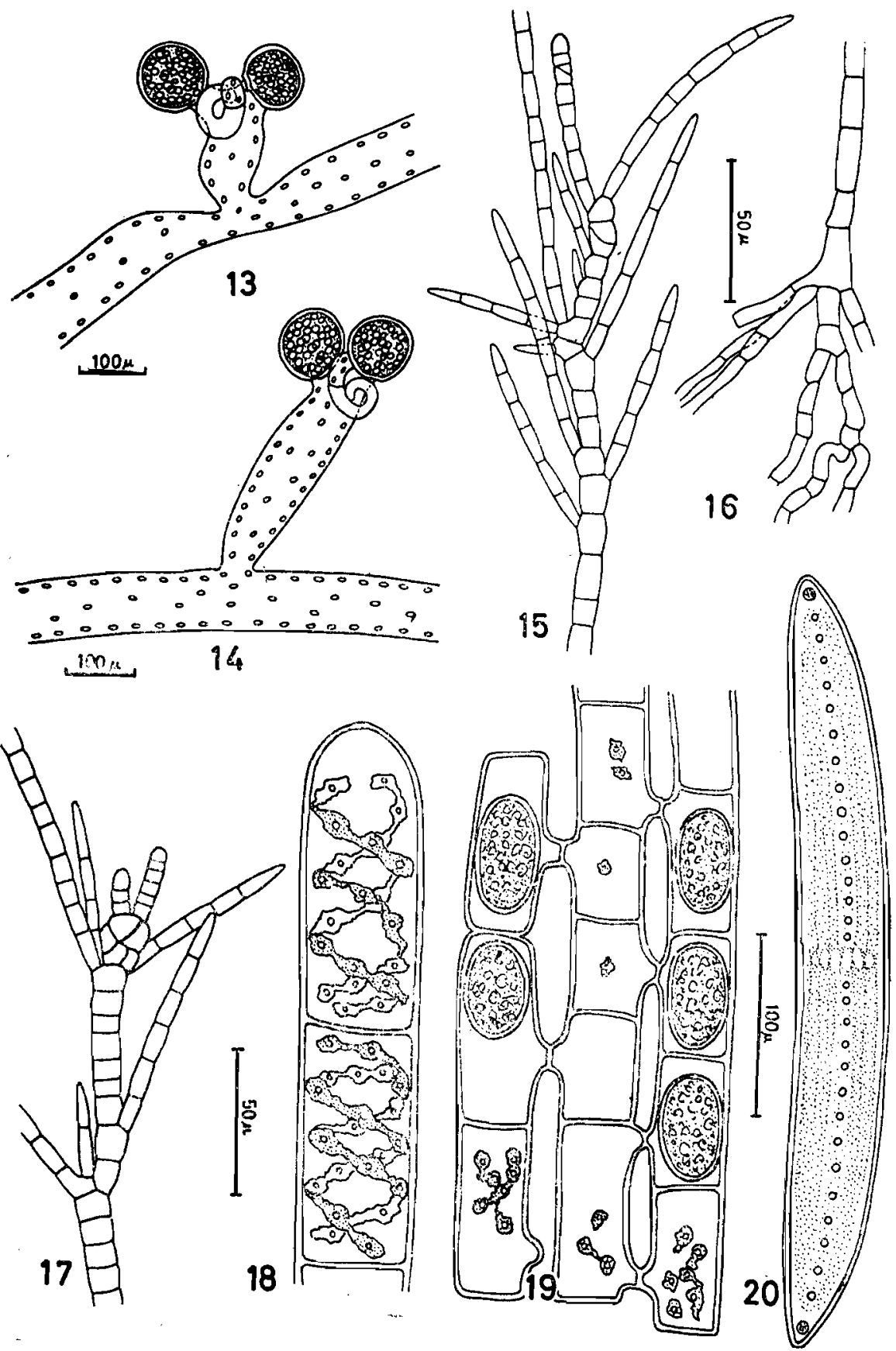

Figs. 13 y 14 Vaucheria geminato (Vaucher) DC., porción vegetativa de un filamento con ramas fértiles, $\infty$ gonios y anteridios característicos. - Figs. 15, 16 y 17 Stigeoclonium lubricum (Dill.) Kützing. (Figs. 15 y 17) Detalle de la parte terminal de un filamento principal y ramificacones. (Fig. 16) Porción rizoidal.— Figs. 18 y 19 Spirogyra decimina (Müller) Kützing. (Fig. 18) Porción terminal de un filamento: características de las células vegetativas. (Fig. 19) Conjugación escaleriforme entre los gametangios de un filamento central y los de dos laterales.- Fig 20 Closterium pseudolunula Borge. 

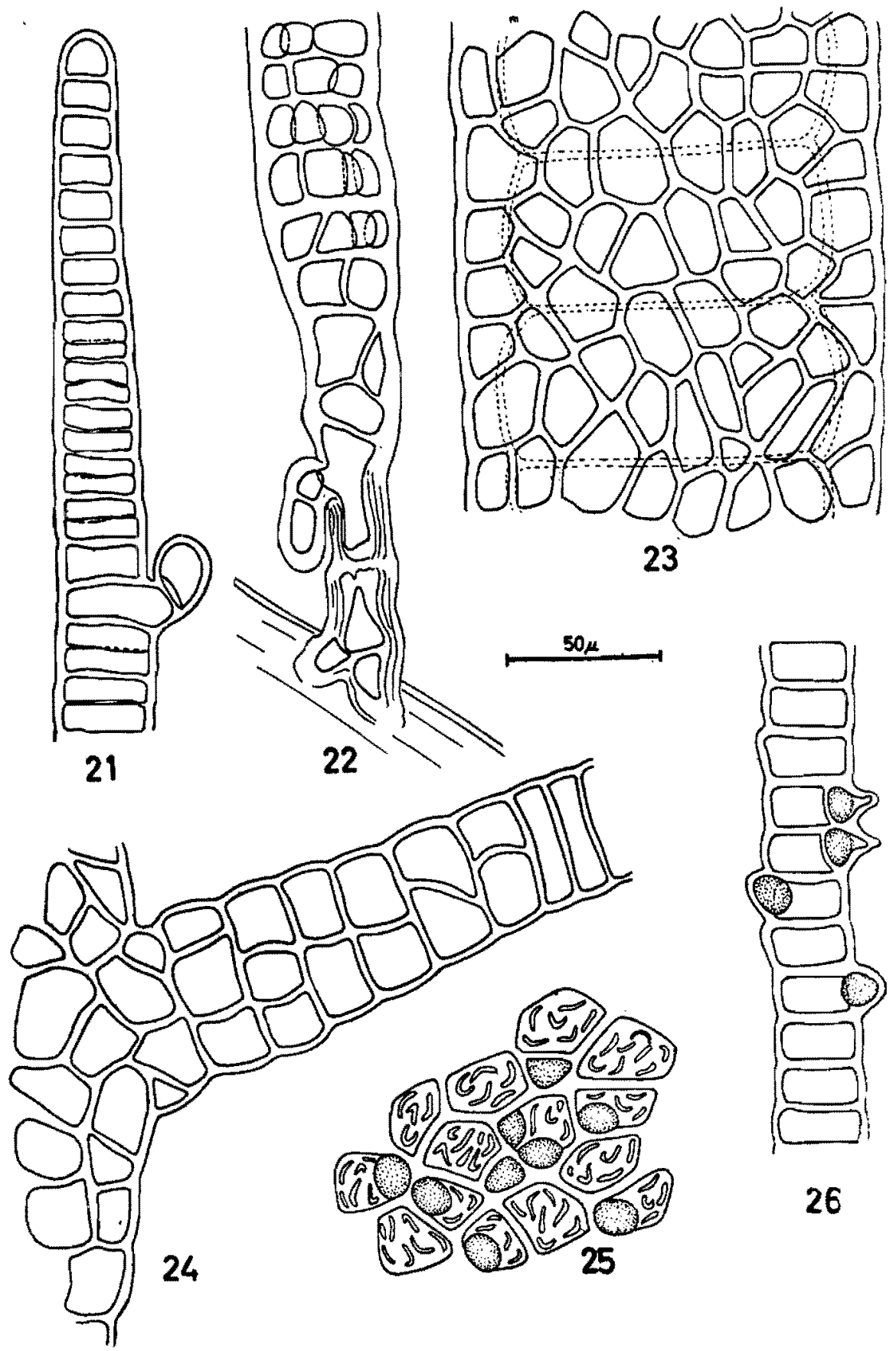

Figs. 21, 22, 23, 24, 25 y 26 Compsopogon coeruleus (Balbis) Montogne. (Fig. 21) Coracterística uniseriada de la porción terminal de una roma juvenil. (Fig. 22) Detalle de la porción rizoidal. (Fig. 23) Porción media en un individuo adulto. (Fig. 24) Parte proximal de una rama parcialmente pluriseriada. (Fig. 25) Características de las células, los cromatóforos y monosporangios. (Fig 26) Porción de una rama uniseriada con monosporangios, dos de ellos en una fase previa a la liberación de las monosporas. 


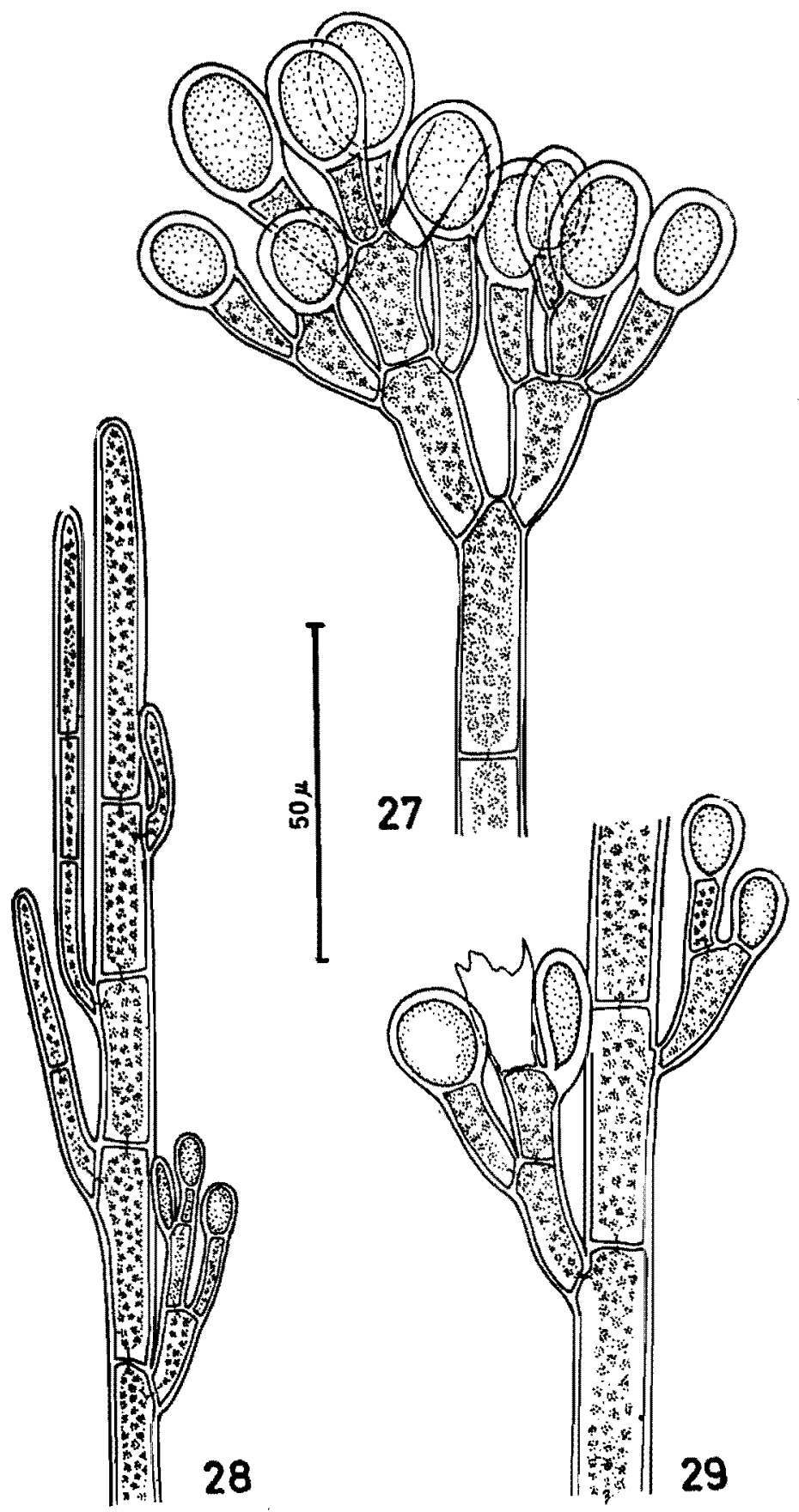

Figs. 27, 28 y 29 Rhodochorton venezuelensis D'Locoste et Gonesan. (Fig. 27) Rama lateral con monosporangios terminales. (Fig. 28) Parte terminal de un filamento principal: características de las células vegetativas y las ramíficaciones. (Fig. 29) Porción media de un filamento principal con ramas laterales y monosporangios. 

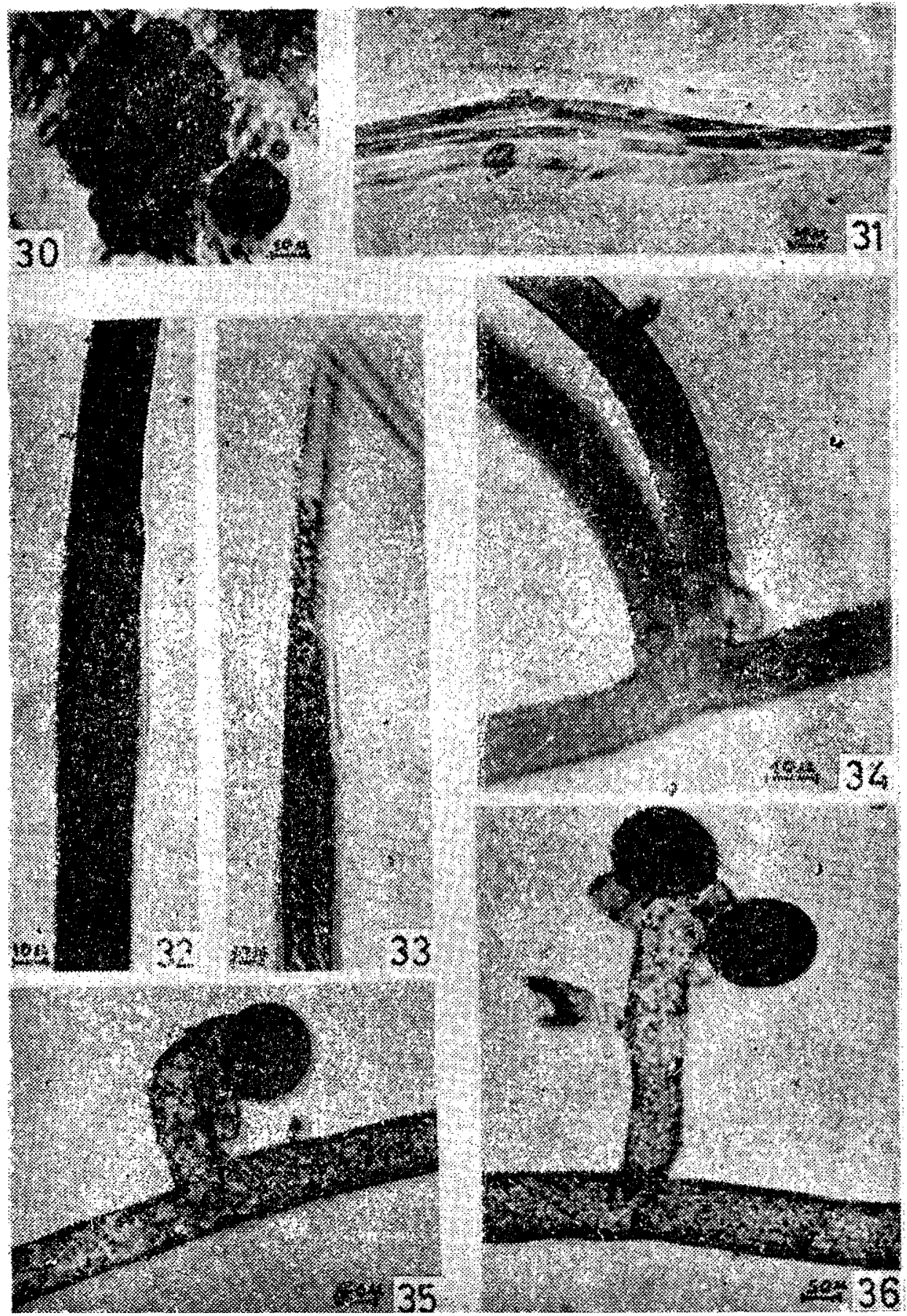

Fig. 30 Dermocarpa versicalor (Borzi) Geitler. Individuos gregorios, epifitos en Rhodochorton yenezuelensis. - Fig. 31 Microcoleus chthonoplastes (Mertens) Thuret. Parte terminal de un filomento-- Figs. 32 y 33 Dichothrix baueriana (Grun.) Born. et Flah. Características de la porción media y terminal de un filomento, respectivomente-_- Fig. 34 Seytonemo haffmannii Agordh ex Gomont et Flah. Detalle de la ramificación.- Figs. 35 y 36 Vaucheria geminata (Voucher) DC. Coracteristicos de las ramas fértiles, oogonio $y$ onteridios. 

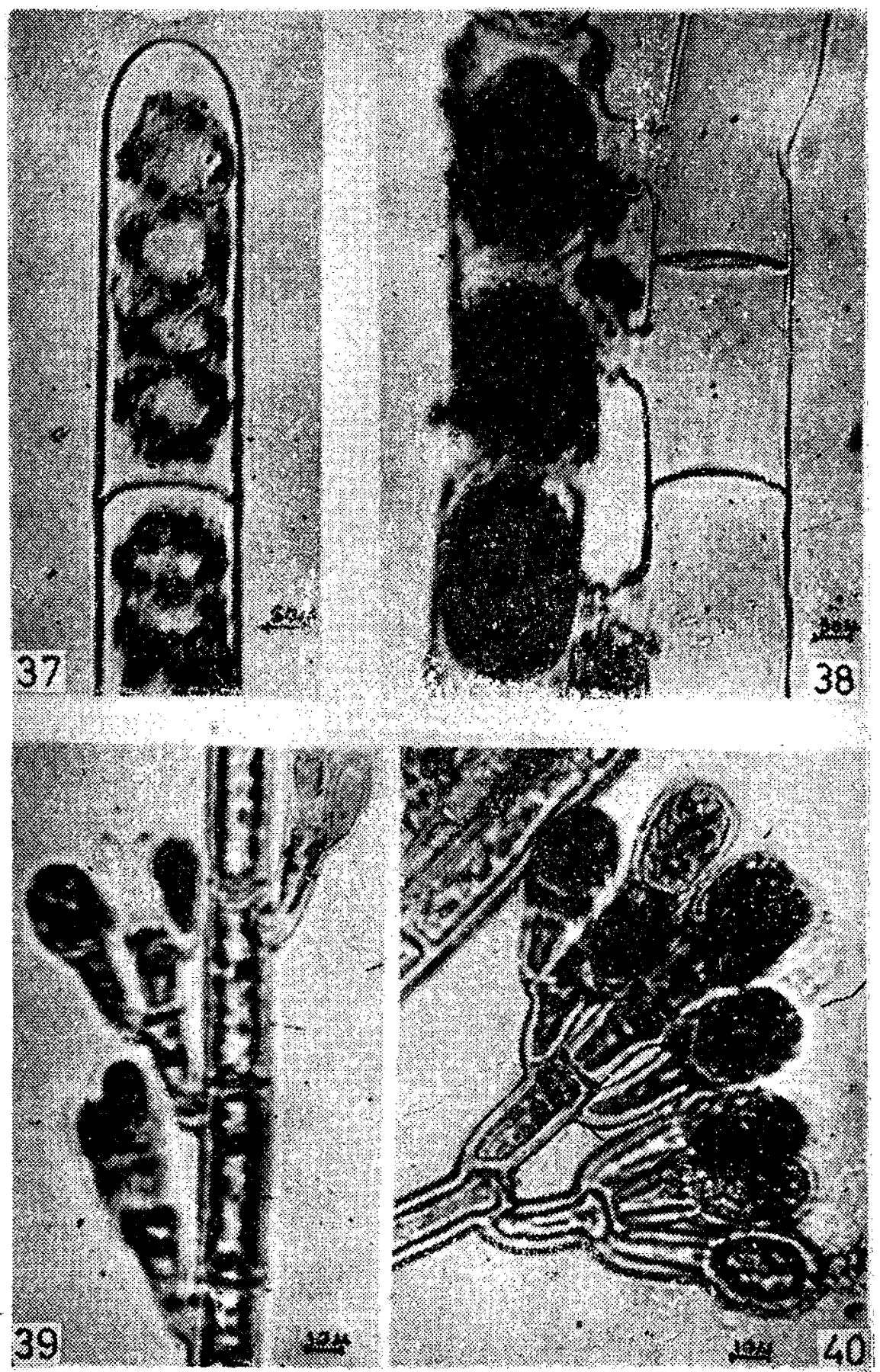

Figs. 37 y 38 Spirogyra decimina (Müller) Kützing. Célula terminal de un filomento y conjugoción escaleriforme, respectivamente, - Figs. 39 y 40 Rhodochoston venezuelensis D'Locoste et Goneson. Porción media de un filomento principol, romificaciones y monos. porangios terminales. 